\title{
MAXIMAL MONOTONE MODEL WITH DELAY TERM OF CONVOLUTION
}

\author{
CLAUDE-HENRI LAMARQUE, JÉRÔME BASTIEN, AND MATTHIEU HOLLAND
}

Received 10 February 2004

Mechanical models are governed either by partial differential equations with boundary conditions and initial conditions (e.g., in the frame of continuum mechanics) or by ordinary differential equations (e.g., after discretization via Galerkin procedure or directly from the model description) with the initial conditions. In order to study dynamical behavior of mechanical systems with a finite number of degrees of freedom including nonsmooth terms (e.g., friction), we consider here problems governed by differential inclusions. To describe effects of particular constitutive laws, we add a delay term. In contrast to previous papers, we introduce delay via a Volterra kernel. We provide existence and uniqueness results by using an Euler implicit numerical scheme; then convergence with its order is established. A few numerical examples are given.

\section{Introduction}

Differential inclusions are often used for modeling of nonsmooth terms occurring from applied sciences: for example, friction $[1,2,3]$ and impacts [6, 9]. Mathematical frames have been extensively studied since the pioneering frameworks by Brézis [5]. Recently we have considered mechanical systems including nonsmooth terms of friction type and we have investigated models and also we have processed identification of models $[1,2,3,4$, 10]. Models have included nonsmooth terms and delay terms [8]. Mathematical models have been obtained and analyzed numerically. But only simple models for the delay term have been studied, involving the particular form $G(u(t-\tau))$ inserted in the evolution equation: that is, only one delay time $\tau$ or a finite number of delay times have been taken into account. Here we extend all the results of reference [8] to more general delay terms of Volterra form (see [7]).

The paper is organized as follows. In Section 2, the models are described. In Section 3, theoretical results are given: uniqueness, existence, and convergence of the numerical scheme. In Section 4, numerical analysis is briefly studied and a few numerical examples are given. 


\section{Description of models}

Let $n$ be an integer. Let $H=\mathbb{R}^{n}$ equipped with the usual scalar product denoted by $\langle\cdot, \cdot\rangle$ and its norm denoted by $|\cdot|, T$ and $\tau$ two strictly positive numbers, and $A$ a maximal monotone multivalued operator on $\mathbb{R}^{n}$, with nonempty domain denoted by $D(A)$. We assume now that $f$ is a function from $[0, T] \times \mathbb{R}^{n}$ to $\mathbb{R}^{n}$, Lipschitz-continuous with respect to its second argument:

$$
\exists L \geq 0, \forall t \in[0, T], \forall x_{1}, x_{2} \in \mathbb{R}^{n}, \quad\left|f\left(t, x_{1}\right)-f\left(t, x_{2}\right)\right| \leq L\left|x_{1}-x_{2}\right|,
$$

and whose derivative has the following property:

$$
\forall R \geq 0, \quad \Phi(R)=\sup \left\{\left\|\frac{\partial f}{\partial t}(\cdot, v)\right\|_{L^{2}\left(0, T ; \mathbb{R}^{n}\right)}:\|v\|_{L^{2}\left(0, T ; \mathbb{R}^{n}\right)} \leq R\right\}<+\infty .
$$

Let $\mathcal{M}_{n}(\mathbb{R})$ be the set of real square matrices of size $n$. Let $\mathscr{H}$ be a function from $[0, \tau]$ to $\mathcal{M}_{n}(\mathbb{R})$ such that each component $\mathscr{H}_{i j}$ of $\mathscr{H}$ satisfies

$$
\forall i, j \in\{1, \ldots, n\}, \quad \mathscr{H}_{i j} \in H^{1}(0, \tau)
$$

Let $z$ be a function such that

$$
\begin{gathered}
z \in H^{1}\left(-\tau, 0 ; \mathbb{R}^{n}\right), \\
z(0) \in D(A) .
\end{gathered}
$$

Applications arising from several fields, as explained in Section 1, lead to systems that are described by the following general mathematical model: we study functions $u$ from $[-\tau, T]$ to $\mathbb{R}^{n}$ satisfying the differential inclusion with delay term of convolution:

$$
\dot{u}(t)+A(u(t))+f(t, u(t))+\int_{0}^{\tau} \mathscr{H}(s) u(t-s) d s \ni 0, \quad \text { a.e. on }(0, T),
$$

with initial condition

$$
\forall t \in[-\tau, 0], \quad u(t)=z(t)
$$

\section{Theoretical results: uniqueness, existence, and convergence of the numerical scheme}

As in [2], we study differential inclusion (2.2), by introducing a numerical scheme. Then, we establish uniform inequalities of the discrete values of solutions of the numerical scheme and by passing to the limit, we obtain the existence of problem (2.2). We prove also the uniqueness of the solution of this problem. We conclude the study by giving two results of order of convergence.

All results of this section are very close to theoretical results of [2]. So, to simplify this paper, we only give the main theoretical results in this section; moreover, we assume without loss of generality that $n=1$ and then $H=\mathbb{R}$.

For all this section, we set for all functions $u \in L^{2}(-\tau, T)$,

$$
\forall s \in[0, T], \quad \mathscr{L}(s, u)=\int_{0}^{\tau} \mathscr{H}(x) u(s-x) d x .
$$


3.1. Uniqueness. Before proving uniqueness, we give the following lemma.

Lemma 3.1. If $\mathscr{H}$ belongs to $L^{2}(0, \tau)$, then for all $u_{1}, u_{2} \in L^{2}(-\tau, T)$,

$$
\forall t \in[0, T], \quad \int_{0}^{t}\left(\mathscr{L}\left(s, u_{1}\right)-\mathscr{L}\left(s, u_{2}\right)\right)^{2} d s \leq 3 T\|\mathscr{H}\|_{L^{2}(0, \tau)}^{2} \int_{-\tau}^{t}\left(u_{1}(s)-u_{2}(s)\right)^{2} d s .
$$

Proof. We use the definition (3.1) of $\mathscr{L}$. We obtain the desired result by the inequality

$$
\begin{gathered}
\int_{0}^{t} \int_{s-\tau}^{s}\left(u_{1}(x)-u_{2}(x)\right)^{2} d x d s \\
\leq T\|\mathscr{H}\|_{L^{2}(0, \tau)}^{2}\left(\int_{0}^{t}\left(u_{1}(s)-u_{2}(s)\right)^{2}-\left(u_{1}(s-\tau)-u_{2}(s-\tau)\right)^{2} d s\right. \\
\left.\quad+\int_{t-\tau}^{t}\left(u_{1}(x)-u_{2}(x)\right)^{2} d x\right) .
\end{gathered}
$$

Proposition 3.2. Let $A$ be a maximal monotone multivalued operator from $\mathbb{R}^{n}$ to $\mathbb{R}^{n}$. Under assumptions (2.1), there exists at most one function $u$ belonging to $H^{1}\left(-\tau, T ; \mathbb{R}^{n}\right)$ and satisfying (2.2).

Proof. This proposition is very similar to [2, Proposition 2.1], based on the discrete Gronwall's lemma. As for the proof of [2, Proposition 2.1], we have, since $u_{1}$ and $u_{2}$ satisfy (2.2a)

$$
\begin{aligned}
\forall t \in[0, T], \quad \frac{1}{2}\left(u_{1}(t)-u_{2}(t)\right)^{2} \leq & \left(L+\frac{1}{2}\right) \int_{0}^{t}\left(u_{1}(s)-u_{2}(s)\right)^{2} d s \\
& +\frac{1}{2} \int_{0}^{t}\left(\mathscr{L}\left(s, u_{1}\right)-\mathscr{L}\left(s, u_{2}\right)\right)^{2} d s
\end{aligned}
$$

Thanks to Lemma 3.1 and the initial condition (2.2b), we obtain

$$
\frac{1}{2}\left(u_{1}(t)-u_{2}(t)\right)^{2} \leq\left(L+\frac{3}{2} T\|\mathscr{H}\|_{L^{2}(0, \tau)}^{2}+\frac{1}{2}\right) \int_{0}^{t}\left(u_{1}(s)-u_{2}(s)\right)^{2} d s .
$$

By using Gronwall's lemma, we can conclude that $v_{1}-v_{2}=0$.

3.2. The scheme. As in [2], we discretize problem (2.2) by using an implicit Euler scheme. As in [8], we assume without loss of generality that there exists $Q \in \mathbb{N}^{*}$ such that

$$
Q \tau=T
$$

Let $N$ be an integer; we set

$$
M=Q N
$$

and we consider the time-step

$$
h=\frac{\tau}{N} \text {. }
$$


440 Maximal monotone model with delay term of convolution

For all $p \in\{-N, \ldots, M\}$, define $t_{p}=p h$ and let $U^{p}$ be the solution of the numerical scheme:

$$
\begin{gathered}
\forall p \in\{0, \ldots, M-1\}, \quad \frac{U^{p+1}-U^{p}}{h}+A\left(U^{p+1}\right)+f\left(t_{p}, U^{p}\right)+W^{p} \ni 0, \\
\forall p \in\{-N, \ldots, 0\}, \quad U^{p}=z(p h) .
\end{gathered}
$$

Here, $W^{p}$ is an approximation of $\int_{0}^{\tau} \mathscr{H}(s) u(t-s) d s$ at times $t_{p}$. We denote by $u_{h}$ the linear interpolation of the $U^{p}$ 's at $t_{p}$. We choose the integration rectangle rule:

$$
W^{p}=h \sum_{l=0}^{N-1} \mathscr{H}(l h) u_{h}\left(t_{p}-l h\right) .
$$

So, we can rewrite numerical scheme (3.9), (3.10), and (3.11) under the form

$$
\begin{gathered}
\forall p \in\{-N, \ldots, 0\}, \quad U^{p}=z(p h), \\
\forall p \in\{0, \ldots, M-1\}, \quad W^{p}=h \sum_{l=0}^{N-1} \mathcal{H}(l h) U^{p-l}, \\
\forall p \in\{0, \ldots, M-1\}, \quad \frac{U^{p+1}-U^{p}}{h}+A\left(U^{p+1}\right)+f\left(t_{p}, U^{p}\right)+W^{p} \ni 0 .
\end{gathered}
$$

This scheme possesses a unique solution: indeed, according to Brézis [5], $A$ is maximal monotone and then, for all $\lambda>0$, the operator $(I+\lambda A)^{-1}$ is defined on all of $\mathbb{R}^{n}$ and single-valued from $\mathbb{R}^{n}$ to $\mathbb{R}^{n}$. Thus, $(3.12 \mathrm{c})$ is equivalent to

$$
\forall p \in\{0, \ldots, M-1\}, \quad U^{p+1}=(I+h A)^{-1}\left(-h\left(f\left(t_{p}, U^{p}\right)+W^{p}\right)+U^{p}\right) .
$$

We set

$$
u_{0}=z(0)
$$

The values $U^{p}$ are uniformly bounded with $h$.

Lemma 3.3. We assume that (2.1) hold. There exists a constant $C_{1}$ such that for all $N \in \mathbb{N}^{*}$,

$$
\begin{gathered}
\left\|u_{h}-u_{0}\right\|_{C^{0}\left([-\tau, T], \mathbb{R}^{n}\right)} \leq C_{1}, \\
\left\|\dot{u}_{h}\right\|_{L^{\infty}\left(-\tau, T ; \mathbb{R}^{n}\right)} \leq C_{1} .
\end{gathered}
$$

Before proving Lemma 3.3, give a lemma, based on the discrete Gronwall lemma.

Lemma 3.4. Let $P$ be an integer, $\Lambda$ and $h$ two nonnegative numbers, and positive reals $\left(a_{j}\right)_{j \in\{0, \ldots, P\}}$ satisfying

$$
\forall j \in\{1, \ldots, P\}, \quad a_{j} \leq \Lambda\left(1+h \sum_{k=0}^{j-1} a_{k}\right) .
$$


Then,

$$
\forall j \in\{0, \ldots, P\}, \quad a_{j} \leq \Lambda\left(h a_{0}+1\right) e^{\Lambda(j-1) h} .
$$

Proof. We set for all $p \in\{0, \ldots, P\}$,

$$
S_{p}=\sum_{l=0}^{p} a_{l}
$$

Inequality (3.17) can then be rewritten under the form

$$
\forall j \in\{1, \ldots, P\}, \quad S_{j} \leq(1+\Lambda h) S_{j-1}+\Lambda .
$$

According to the discrete Gronwall lemma, we then have

$$
\begin{aligned}
\forall j \in\{1, \ldots, P\}, \quad S_{j} & \leq S_{0} e^{\Lambda j h}+\Lambda \sum_{k=0}^{j-1} e^{\Lambda k h} \\
& =S_{0} e^{\Lambda j h}+\Lambda \frac{e^{\Lambda j h}-1}{e^{\Lambda h}-1} \\
& \leq S_{0} e^{\Lambda j h}+\frac{e^{\Lambda j h}-1}{h} .
\end{aligned}
$$

So, we have

$$
h S_{j} \leq h S_{0} e^{\Lambda j h}+e^{\Lambda j h}-1 .
$$

From (3.17), we can deduce

$$
\begin{aligned}
\forall j \in\{1, \ldots, P\}, \quad a_{j} & \leq \Lambda+\Lambda\left(h S_{0} e^{\Lambda(j-1) h}+e^{\Lambda(j-1) h}-1\right) \\
& \leq \Lambda\left(h a_{0}+1\right) e^{\Lambda(j-1) h} .
\end{aligned}
$$

Proof of Lemma 3.3. As in [2, Lemmas 2.2 and 2.4], we prove that there exist $\mathscr{C}_{1}$ and $w \in \mathbb{R}^{n}$ such that

$$
\forall p \in\{1, \ldots, M\}, \quad\left(U^{p}-u_{0}\right)^{2} \leq h \mathscr{b}_{1} \sum_{k=0}^{p-1}\left(\left(f\left(t_{k}, u_{0}\right)-f\left(0, u_{0}\right)+w\right)^{2}+\left(W^{k}\right)^{2}\right) .
$$

From assumption (2.1b), we can deduce that the first sum of this inequality is uniformly bounded with $h$. Then, there exists $\mathscr{C}_{2}$ such that

$$
\forall p \in\{1, \ldots, M\}, \quad\left(U^{p}-u_{0}\right)^{2} \leq \mathscr{C}_{2}\left(1+h \sum_{k=0}^{p-1}\left(W^{k}\right)^{2}\right) .
$$


442 Maximal monotone model with delay term of convolution

By using the definition of $W^{k}$, we have, thanks to the Cauchy-Schwarz inequality,

$$
\sum_{k=0}^{p-1}\left(W^{k}\right)^{2} \leq h \sum_{k=0}^{p-1}\left(\sum_{l=0}^{N-1} h \mathscr{H}^{2}(l h) \sum_{l=0}^{N-1}\left(U^{k-l}\right)^{2}\right)
$$

Denote by $\mathscr{H}_{h}$ the piecewise function equal to $H(l p)$ on each interval $\left[t_{p}, t_{p+1}[\right.$ of $[0, \tau]$. So, we can write

$$
\sum_{k=0}^{p-1}\left(W^{k}\right)^{2} \leq h\left\|\mathscr{H}_{h}\right\|_{L^{2}(0, \tau)}^{2} \sum_{k=0}^{p-1} \sum_{l=0}^{N-1}\left(U^{k-l}\right)^{2}
$$

Since

$$
\forall k \in\{0, \ldots, p-1\}, \forall l \in\{0, \ldots, N-1\}, \quad-N \leq k-l \leq p-1,
$$

we have

$$
\sum_{k=0}^{p-1} \sum_{l=0}^{N-1}\left(U^{k-l}\right)^{2} \leq \sum_{k=0}^{p-1} \sum_{r=-N}^{p-1}\left(U^{r}\right)^{2} \leq M \sum_{r=-N}^{p-1}\left(U^{r}\right)^{2}
$$

According to (3.25) and (3.27), we then have

$$
\forall p \in\{1, \ldots, M\}, \quad\left(U^{p}-u_{0}\right)^{2} \leq \mathscr{C}_{2}\left(1+h^{2} M\left\|\mathscr{H}_{h}\right\|_{L^{2}(0, \tau)}^{2} \sum_{r=-N}^{p-1}\left(U^{r}\right)^{2}\right) .
$$

Since $\mathscr{H} \in L^{2}(0, \tau)$, we have

$$
\sup _{h>0}\left\|\mathscr{H}_{h}\right\|_{L^{2}(0, \tau)}^{2}<+\infty
$$

and according to (3.30), there exists $\mathscr{C}_{3}$ such that for all $h$,

$$
\forall p \in\{1, \ldots, M\}, \quad\left(U^{p}-u_{0}\right)^{2} \leq \mathscr{C}_{3}\left(1+h \sum_{r=-N}^{p-1}\left(U^{r}\right)^{2}\right)
$$


which implies there exists $\mathscr{C}_{4}$ such that

$$
\forall p \in\{1, \ldots, M\}, \quad\left(U^{p}-u_{0}\right)^{2} \leq \mathscr{C}_{4}\left(1+h \sum_{r=-N}^{p-1}\left(U^{r}-u_{0}\right)^{2}\right) .
$$

Since

$$
\forall p \in\{1, \ldots, M\}, \quad \sum_{r=-N}^{p-1}\left(U^{r}-u_{0}\right)^{2}=\sum_{r=-N}^{-1}\left(U^{r}-u_{0}\right)^{2}+\sum_{r=0}^{p-1}\left(U^{r}-u_{0}\right)^{2},
$$

thanks to initial condition (2.2b) and assumption (2.1d), we deduce from (3.33) that there exists $\mathscr{C}_{5}$ such that

$$
\forall p \in\{1, \ldots, M\}, \quad\left(U^{p}-u_{0}\right)^{2} \leq \mathscr{C}_{5}\left(1+h \sum_{r=0}^{p-1}\left(U^{r}-u_{0}\right)^{2}\right) .
$$

Thanks to Lemma 3.4 applied with $a_{j}=\left(U^{j}-u_{0}\right)^{2}$ and $\Lambda=\mathscr{C}_{5}$, we deduce from (3.35) that

$$
\forall j \in\{0, \ldots, M\}, \quad\left(U^{j}-u_{0}\right)^{2} \leq \mathscr{C}_{5} e^{\mathscr{C}_{5}(j-1) h} \leq \mathscr{C}_{5} e^{\mathscr{C}_{5} T} .
$$

So, the conclusion (3.15) is obtained.

We now prove (3.16), as in [2, Lemma 2.2]. Denote by $V^{p}$ the discrete speed defined by

$$
\forall p \in\{0, \ldots, M-1\}, \quad V^{p}=\frac{U^{p+1}-U^{p}}{h} .
$$

As in [2], we obtain

$$
\begin{aligned}
\forall p \in\{1, \ldots, M-1\}, \quad\left(V^{p}-V^{p-1}\right) V^{p} \leq & \left(-f\left(t_{p}, U^{p}\right)+f\left(t_{p-1}, U^{p-1}\right)\right) V^{p} \\
& +\left(W^{p}-W^{p-1}\right) V^{p}
\end{aligned}
$$

and then

$$
\begin{aligned}
& \forall p \in\{1, \ldots, M-1\}, \\
& \quad\left(V^{p}\right)^{2} \leq\left(V^{p-1}\right)^{2}+2\left|V^{p}\right|\left(L h\left|V^{p-1}\right|+\left|\delta_{p}\right|+\left|W^{p}-W^{p-1}\right|\right),
\end{aligned}
$$

where

$$
\delta_{p}=-f\left(t_{p}, U^{p-1}\right)+f\left(t_{p-1}, U^{p-1}\right) .
$$

We obtain

$$
\begin{aligned}
& \forall p \in\{1, \ldots, M-1\}, \\
& \quad(1-2 h-L h)\left(V^{p}\right)^{2} \leq(1+L h)\left(V^{p-1}\right)^{2}+\frac{\delta_{p}^{2}}{h}+\frac{\left(W^{p}-W^{p-1}\right)^{2}}{h} .
\end{aligned}
$$


444 Maximal monotone model with delay term of convolution

If $h$ is small enough,

$$
(1-2 h-L h)^{-1}(1+L h) \leq 1+\mathscr{C}_{6} h
$$

where $\mathscr{C}_{6}$ is not depending on $h$. We also have

$$
(1-2 h-L h)^{-1} \leq 2
$$

So, we obtain

$$
\forall p \in\{1, \ldots, M-1\}, \quad\left(V^{p}\right)^{2} \leq\left(1+\mathscr{C}_{6} h\right)\left(V^{p-1}\right)^{2}+2 \frac{\delta_{p}^{2}}{h}+2 \frac{\left(W^{p}-W^{p-1}\right)^{2}}{h},
$$

and thanks to the discrete Gronwall lemma,

$$
\left(V^{p}\right)^{2} \leq e^{\mathscr{C}_{6} T}\left(\left(V^{0}\right)^{2}+\frac{2}{h} \sum_{k=1}^{M-1} \delta_{k}^{2}+\frac{2}{h} \sum_{k=1}^{M-1}\left(W^{k}-W^{k-1}\right)^{2}\right)
$$

As in [2], we prove that $V^{0}$ and $(1 / h) \sum_{k=1}^{M-1} \delta_{k}^{2}$ are uniformly bounded with $h$. To conclude, we prove that there exists $\mathscr{C}_{7}$ such that for all $h$,

$$
\frac{1}{h} \sum_{k=1}^{M-1}\left(W^{k}-W^{k-1}\right)^{2} \leq \mathscr{C}_{7}
$$

By using the Cauchy-Schwarz inequality and (3.15), we write successively

$$
\begin{aligned}
& \left|W^{k}-W^{k-1}\right| \\
& =h\left|\sum_{l=0}^{N-1} \mathscr{H}(h l) U^{k-l}-\sum_{l=0}^{N-1} \mathscr{H}(h l) U^{k-1-l}\right| \\
& \leq 2 h C_{1}\|\mathscr{H}\|_{C^{0}([0, \tau])}+h \sum_{l=1}^{N-1}|\mathscr{H}(h l)-\mathscr{H}(h l-h)|\left|U^{k-l}\right| \\
& \leq 2 h C_{1}\|\mathscr{H}\|_{C^{0}([0, \tau])}+h\left(\sum_{l=1}^{N-1}(\mathscr{H}(h l)-\mathscr{H}(h l-h))^{2} \sum_{l=1}^{N-1}\left(U^{k-l}\right)^{2}\right)^{1 / 2} \\
& \leq \mathscr{C}_{8}\left(h+\sqrt{h}\left(\sum_{l=1}^{N-1}(\mathscr{H}(h l)-\mathscr{H}(h l-h))^{2}\right)^{1 / 2}\right) \\
& \leq \mathscr{C}_{8}\left(h+\sqrt{h}\left(\sum_{l=1}^{N-1}\left(\int_{t_{l}}^{t_{l+1}} \frac{d \mathscr{H}}{d t}(s) d s\right)^{2}\right)^{1 / 2}\right) \\
& \leq h \mathscr{C}_{8}\left(1+\left(\int_{0}^{\tau}\left(\frac{d \mathscr{H}}{d t}(s)\right)^{2} d s\right)^{1 / 2}\right) \\
& \quad=h \mathscr{C}_{8}\left(1+\|\mathscr{H}\|_{L^{2}(0, \tau)}\right),
\end{aligned}
$$


and by summation

$$
\frac{1}{h} \sum_{k=1}^{M-1}\left(W^{k}-W^{k-1}\right)^{2} \leq T^{\mathscr{C}}{ }_{8}^{2}\left(1+\|\dot{\mathcal{H}}\|_{L^{2}(0, \tau)}\right)^{2} .
$$

Then, we can prove the following.

Proposition 3.5. If assumptions (2.1) hold, then there exists a constant $C_{2}$ such that for all $h, k>0$,

$$
\forall t \in[-\tau, T], \quad\left|u_{h}(t)-u_{k}(t)\right| \leq C_{2} \sqrt{h+k}
$$

As in [2], we might think that the estimates obtained at Lemma 3.3 are sufficient for passing to the limit; define piecewise constant functions: for all $p \in\{-N, \ldots, M-1\}$,

$$
\begin{aligned}
& v_{h}(t)=U^{p+1} \quad \text { on }\left[t_{p}, t_{p+1}[,\right. \\
& \tilde{v}_{h}(t)=U^{p} \quad \text { on }\left[t_{p}, t_{p+1}[,\right. \\
& f_{h}(t)=f\left(t_{p}, U^{p}\right) \quad \text { on }\left[t_{p}, t_{p+1}[,\right.
\end{aligned}
$$

for all $p \in\{0, \ldots, M-1\}$,

$$
\mathscr{L}_{h}(t)=W^{p} \quad \text { on }\left[t_{p}, t_{p+1}[\right.
$$

for all $p \in\{0, \ldots, N-1\}$,

$$
\mathscr{H}_{h}(t)=\mathscr{H}\left(t_{p}\right) \text { on }\left[t_{p}, t_{p+1}[\right.
$$

and let $u_{h}$ be the piecewise linear interpolation taking the value $U^{p}$ at $t_{p}$. As in [2], we are going to prove that the sequence $\left(u_{h}\right)_{h>0}$ is a Cauchy sequence and we will estimate $\left\|u_{h}-u_{k}\right\|_{L^{\infty}(0, T)}$ and $\left\|u_{h}-u_{k}\right\|_{L^{2}(0, T)}$ in terms of $\sqrt{h+k}$. These estimates depend on a couple a preliminary lemmas which strongly use the regularity assumptions.

LemMA 3.6. If assumptions (2.1) hold, then there exists a constant $\mathscr{Y}_{1}$ such that for all $h>0$,

$$
\left\|u_{h}-v_{h}\right\|_{L^{2}(-\tau, T)}+\left\|u_{h}-\widetilde{v}_{h}\right\|_{L^{2}(-\tau, T)} \leq \mathscr{K}_{1} h .
$$

Proof. The proof is similar to that of [2, Lemma 2.3].

Lemma 3.7. If assumptions (2.1) hold, then there exists a constant $\mathscr{Y}_{2}$ such that for all $h>0$,

$$
\left\|f\left(\cdot, \widetilde{v}_{h}\right)-f_{h}\right\|_{L^{2}(-\tau, T)} \leq \mathscr{K}_{2} h
$$

Proof. The proof is similar to that of [2, Lemma 2.4].

Lemma 3.8. If assumptions (2.1) hold, then there exists a constant $\mathscr{Y}_{3}$ such that for all $h>0$,

$$
\forall t \in[0, T], \quad\left|\int_{0}^{\tau} \mathscr{H}(s) \widetilde{v}_{h}(t-s) d s-\mathscr{L}_{h}(t)\right| \leq \mathscr{K}_{3} h
$$


446 Maximal monotone model with delay term of convolution

Proof. By using the Cauchy-Schwarz inequality, we can write

$$
\left|\int_{0}^{\tau} \mathscr{H}(s) \tilde{v}_{h}(t-s) d s-\mathscr{L}_{h}(t)\right| \leq\left(\int_{0}^{\tau}\left(\widetilde{v}_{h}(t-s)\right)^{2} d s \int_{0}^{\tau}\left(\mathscr{H}(s)-\mathscr{H}_{h}(s)\right)^{2} d s\right)^{1 / 2} .
$$

We then write as in the proof of [2, Lemma 2.4]

$$
\int_{t_{l}}^{t_{l+1}}\left(\mathscr{H}(s)-\mathscr{H}_{h}(s)\right)^{2} d s \leq \frac{h^{2}}{2} \int_{t_{l}}^{t_{l+1}}\left(\frac{d \mathscr{H}}{d t}(s)\right)^{2} d s
$$

and by summing

$$
\int_{0}^{\tau}\left(\mathscr{H}(s)-\mathscr{H}_{h}(s)\right)^{2} d s \leq \frac{h^{2}}{2}\|\dot{H}\|_{L^{2}(0, \tau)}^{2}
$$

which permits us to conclude thanks to (3.15) and (3.58).

Proof of Proposition 3.5. As in the proof of [2, Proposition 2.5], we write that the equations satisfied, respectively, by $u_{h}$ and $u_{k}$ are

$$
\begin{aligned}
& \dot{u}_{h}+A v_{h}+f_{h}+\mathscr{L}_{h} \ni 0, \\
& \dot{u}_{k}+A v_{k}+f_{k}+\mathscr{L}_{k} \ni 0 .
\end{aligned}
$$

We then subtract (3.62) from (3.61), allowing for the usual abuse of notations, and multiply by $v_{h}-v_{k}$. We conclude by using Lemmas 3.6, 3.7, and 3.8.

3.3. Uniqueness and existence. From Proposition 3.5, we can deduce the following.

Proposition 3.9. Assume that (2.1) holds. There exists a unique solution $u$ of (2.2) belonging to $W^{1, \infty}\left(-\tau, T ; \mathbb{R}^{n}\right)$. Moreover, if we denote by $u_{h}$ the approximation defined by (3.12), it holds that

$$
\lim _{h \rightarrow 0^{+}} \max _{t \in[-\tau, T]}\left|u(t)-u_{h}(t)\right|=0 .
$$

Proof. It is very close to the proof of [2, Proposition 2.5]. The uniqueness of the solution is already proved. Thanks to (3.15) and (3.16), we extract a subsequence still denoted by $\left(u_{h}\right)_{h>0}$ which converges in the following sense to the function $u$ :

$$
\begin{array}{ll}
u_{h} \rightarrow u & \text { in } L^{\infty}(-\tau, T) \text { weak } *, \\
u_{h} \rightarrow u & \text { in } L^{2}(-\tau, T) \text { weak, } \\
\dot{u}_{h} \rightarrow \dot{u} & \text { in } L^{\infty}(-\tau, T) \text { weak } *, \\
\dot{u}_{h} \rightarrow \dot{u} & \text { in } L^{2}(-\tau, T) \text { weak. }
\end{array}
$$

We conclude as in [2, Proposition 2.5]. 
3.4. Order of convergence. We have the following two results.

Proposition 3.10. If assumptions (2.1) hold, then the scheme is of order $1 / 2$, that is, there exists a constant $C$ such that

$$
\forall h>0, \quad \max _{t \in[-\tau, T]}\left|u(t)-u_{h}(t)\right| \leq C \sqrt{h}
$$

Proof. As in [2, Proposition 2.6], thanks to estimate (3.49) for $k \rightarrow 0$ and (3.63), we obtain

$$
\max _{t \in[-\tau, T]}\left|u(t)-u_{h}(t)\right| \leq C_{2} \sqrt{h}
$$

We assume now that $K$ is a nonempty closed convex subset of $\mathbb{R}^{n}$ and that $A$ is the subdifferential of the indicatrix of the convex $K$ defined by

$$
\psi_{K}(x)= \begin{cases}0 & \text { if } x \in K \\ +\infty & \text { if } x \notin K\end{cases}
$$

In this particular case, we have

$$
\begin{gathered}
\forall(x, y) \in K \times \mathbb{R}^{n}, \quad y \in \partial \psi_{K}(x) \Longleftrightarrow \forall z \in K, \quad\langle y, x-z\rangle \geq 0, \\
\forall x \notin K, \quad \partial \psi_{K}(x)=\varnothing .
\end{gathered}
$$

Proposition 3.11. Let $K$ be a nonempty closed convex subset of $\mathbb{R}^{n}$ and $A$ the maximal monotone operator $\partial \psi_{K}$. If hypotheses (2.1) hold, then the order of the scheme is one, that is, there exists $C$ such that

$$
\forall h>0, \quad \max _{t \in[-\tau, T]}\left|u(t)-u_{h}(t)\right| \leq C h
$$

Proof. See the proof of [2, Proposition 3.1].

\section{Numerical simulations}

For numerical simulations, we study the following class of example: we consider $b$ and $\alpha$ real constants. $\sigma$ denotes the graph of sign function and let $g \in L^{2}(0, T)$ and $H \in H^{1}(0, \tau)$. We consider a one degree-of-freedom mechanical system governed by the inclusion

$$
\begin{gathered}
\ddot{w}(t)+b w(t)+\int_{0}^{\tau} H(s) w(t-s) d s+\alpha \sigma(\dot{w}(t))+g(t) \ni 0, \quad \text { a.e. on }(0, T), \\
\forall t \in[-\tau, 0], \quad w(t)=z(t)
\end{gathered}
$$


448 Maximal monotone model with delay term of convolution

This model is of the previous general form (2.2) with $n=2$, by setting

$$
\begin{aligned}
f\left(t,\left(\begin{array}{l}
u \\
v
\end{array}\right)\right) & =\left(\begin{array}{c}
-v \\
b u+g(t)
\end{array}\right), \\
A\left(\begin{array}{l}
u \\
v
\end{array}\right) & =\{0\} \times\{\alpha \sigma(v)\}, \\
\mathscr{H}(s) & =\left(\begin{array}{cc}
0 & 0 \\
H(s) & 0
\end{array}\right) .
\end{aligned}
$$

Thanks to these definitions, numerical scheme (3.13) is equivalent to

$$
\begin{gathered}
u^{p+1}=h v^{p}+u^{p} \\
v^{p+1}=(I+h \alpha \sigma)^{-1}\left(-h\left(b u^{p}+g\left(t_{p}\right)+h \sum_{l=0}^{N-1} \mathscr{H}(l h) u^{p-l}\right)+v^{p}\right) .
\end{gathered}
$$

For all the numerical simulations, we choose

$$
b=1, \quad \alpha=1, \quad \tau=1, \quad h=\frac{1}{9991} .
$$

Example 4.1. We choose

$$
\begin{aligned}
& T=10, \quad H(s)=1, \\
& g(t)= \begin{cases}(-t+4) e^{-t}+(-t+1) e^{-t+1}-1, & \text { if } t \in]-\infty, 2[, \\
(-t+4) e^{-t}+(-t+1) e^{-t+1}, & \text { if } t=2, \\
(-t+4) e^{-t}+(-t+1) e^{-t+1}+1, & \text { if } t \in] 2,+\infty[,\end{cases}
\end{aligned}
$$

so that

$$
w(t)=(t-1) e^{-t}
$$

is exact solution on $[-1, T]$. Numerical results are presented in Figure 4.1.

Exact solution and approximated solution are plotted simultaneously. Curves are again superimposed. In this case, derivative $\dot{w}$ possesses one change of sign. 


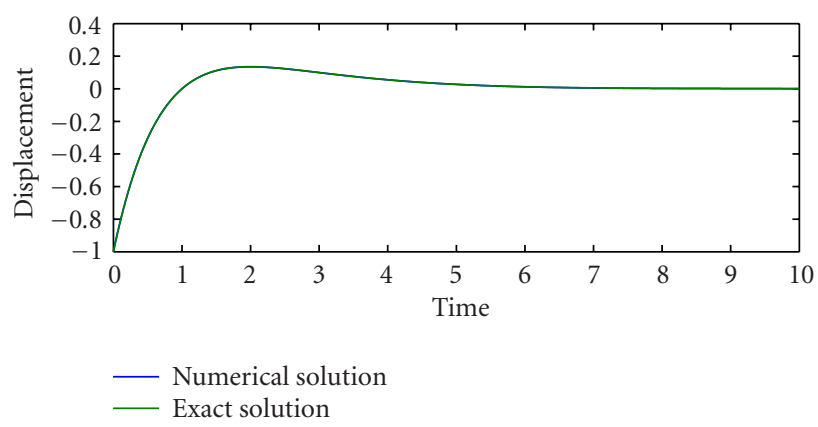

(a)

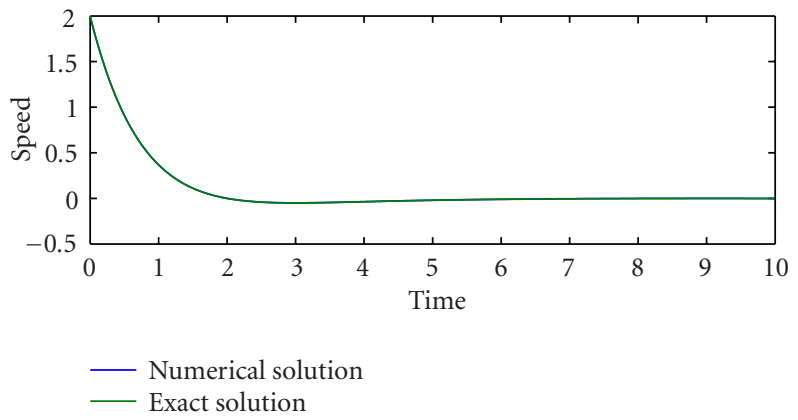

(b)

Figure 4.1. (a) Displacement and (b) speed versus time for Example 4.1.

Example 4.2. We choose $T$ and $H$ defined by (4.6) and

$$
g(t)= \begin{cases}\left(-t^{2}+7 t-4\right) e^{-t}+\left(-t^{2}+t-2\right) e^{-t+1}+1, & \text { if } t \in]-\infty, 1[ \\ \left(-t^{2}+7 t-4\right) e^{-t}+\left(-t^{2}+t-2\right) e^{-t+1}, & \text { if } t=1, \\ \left(-t^{2}+7 t-4\right) e^{-t}+\left(-t^{2}+t-2\right) e^{-t+1}-1, & \text { if } t \in] 1,2[, \\ \left(-t^{2}+7 t-4\right) e^{-t}+\left(-t^{2}+t-2\right) e^{-t+1}, & \text { if } t=2, \\ \left(-t^{2}+7 t-4\right) e^{-t}+\left(-t^{2}+t-2\right) e^{-t+1}+1, & \text { if } t \in] 2,+\infty[\end{cases}
$$

so that

$$
w(t)=\left(t^{2}-t+1\right) e^{-t}
$$

is exact solution on $[-1, T]$. Numerical results are presented in Figure 4.2.

Exact solution and approximated solution are plotted simultaneously. Curves are again superimposed. In this case, derivative $\dot{w}$ changes its sign twice. 


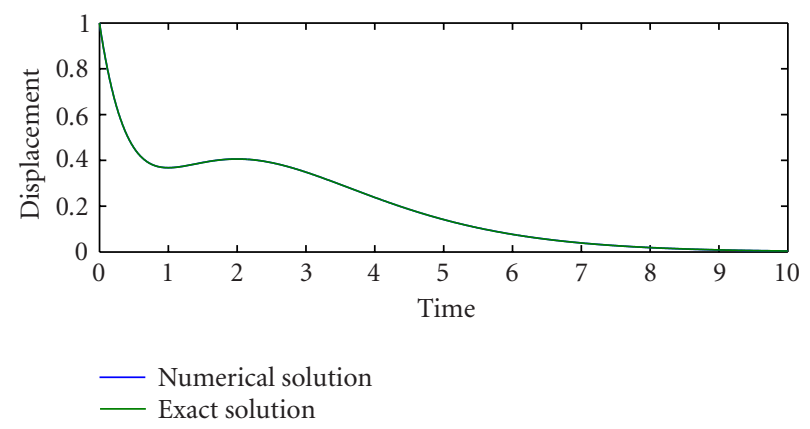

(a)

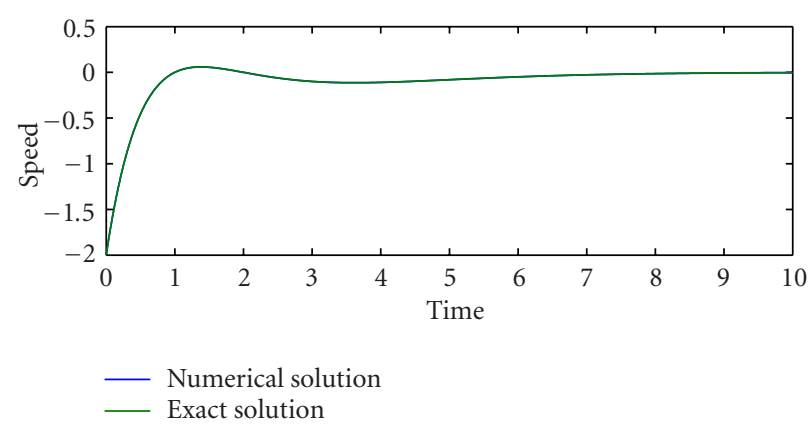

(b)

Figure 4.2. (a) Displacement and (b) speed versus time for Example 4.2.

In order to test numerically the order of the scheme, maximal error between exact solution and approximated solution has been computed for Example 4.2 for 18 values of time steps $1 /(1001+50 k), k=1, \ldots, 18$. In Figure 4.3, a log-log plot is presented. The slope of the straight line corresponds to numerically estimated order of the numerical scheme. A least-square computation provides value 0.993 very close to 1 in this case. In fact, Proposition 3.10 gives an order equal to $1 / 2$. The order one with $A=\sigma$ is an open problem.

Example 4.3. We choose $T=30$ and $H$ defined by

$$
\begin{array}{r}
H(t)=\cos (2 \pi t)+\cos (10 \pi t)+\cos (20 \pi t), \\
g(t)=1,3 \cos (8 t), \quad z(t)=0 .
\end{array}
$$

Numerical results are presented in Figures 4.4 and 4.5. In this case, there are a finite number of static phases (where $\dot{w}$ is vanish) and dynamic phases (where $\dot{w}$ is not vanish). 


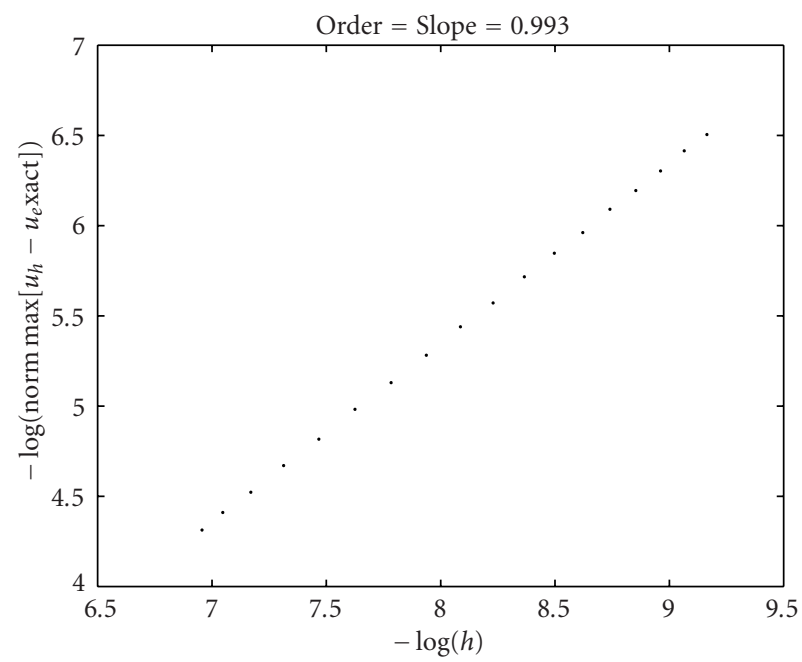

Figure 4.3. Log-log plot of maximal error versus time steps for Example 4.2. Numerical estimation of the order corresponds to the least-square slope of the curve.

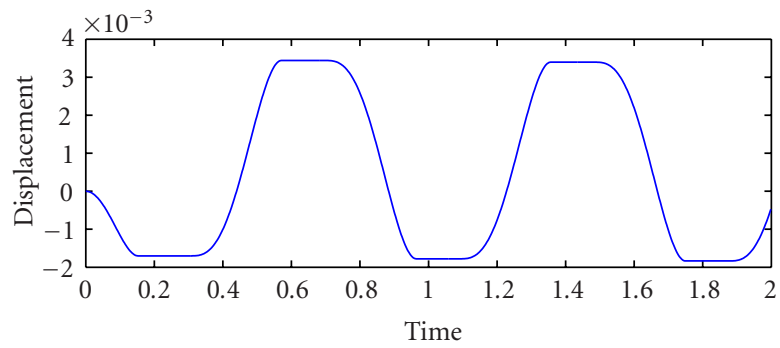

(a)

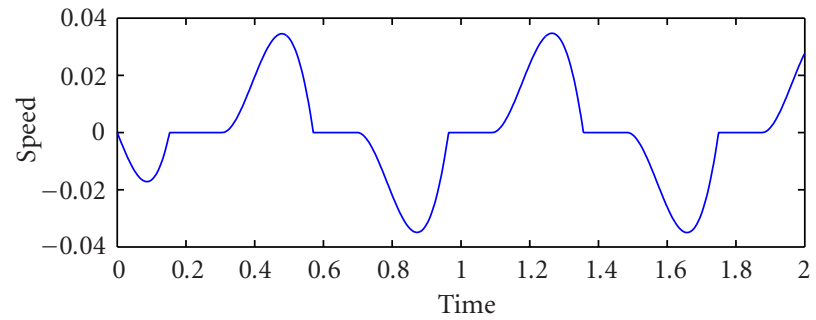

(b)

Figure 4.4. (a) Displacement and (b) speed versus time (on [0,2]) for Example 4.3. 


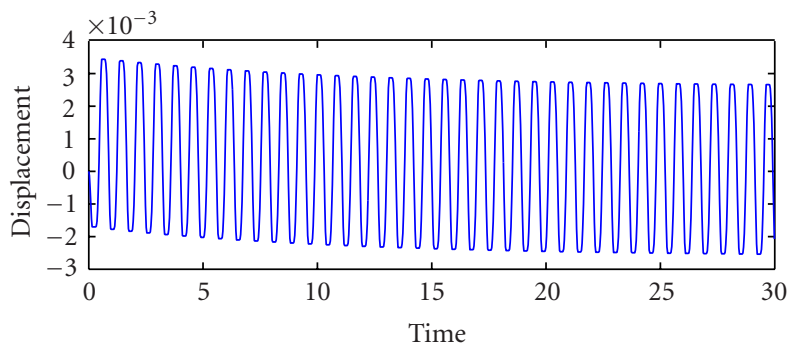

(a)

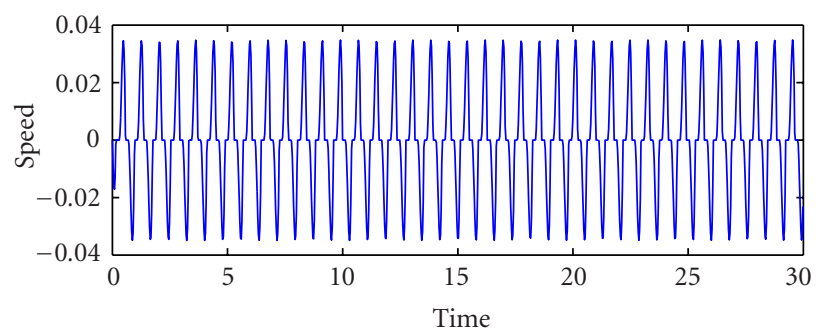

(b)

Figure 4.5. (a) Displacement and (b) speed versus time (on $[0,30]$ ) for Example 4.3.

\section{Conclusions and perspectives}

In this paper, results obtained for differential inclusions with delay term of special form in [8] have been extended to another case where delay terms are provided by Volterra kernel.

Existence and uniqueness have been proved with assumptions commonly verified by models issuing from applications. Numerical analysis has been performed. Theoretical order of convergence is $1 / 2$ in the general case or 1 if $A=\partial \psi_{K}$.

In a future work, we will present a complete proof of the results of Section 3; Moreover, to generalize results of this paper, and of $[2,8]$, as in $[2]$, we will give a frame to study differential problem

$$
\dot{u}(t)+A(u(t))+f(t, u(t))+\mathscr{L}(t, u) \ni 0, \quad \text { a.e. on }(0, T)
$$

with initial condition

$$
\forall t \in[-\tau, 0], \quad u(t)=z(t)
$$

Here, $H$ denotes a Hilbert space, $u$ is a function belonging to $C^{0}([-\tau, T] ; H)$ whose derivative belongs to $L^{\infty}(-\tau, T ; H) . \mathscr{L}$ is the "history" term: it is a function from $[0, T] \times$ $C^{0}([-\tau, T] ; H)$ depending only on $t$ and of values $\{u(s): s \in[-\tau, t]\}$ from $u$. We will 
assume that this term is continuous according to $u$ in the following sense: there exists $\mathscr{C}$ such that for all $u_{1}, u_{2}$ belonging to $C^{0}([-\tau, T] ; H)$,

$$
\forall t \in[0, T], \quad \int_{0}^{t}\left(\mathscr{L}\left(s, u_{1}\right)-\mathscr{L}\left(s, u_{2}\right)\right)^{2} d s \leq \mathscr{C} \int_{-\tau}^{t}\left(u_{1}(s)-u_{2}(s)\right)^{2} d s .
$$

\section{References}

[1] J. Bastien, Étude théorique et numérique d'inclusions différentielles maximales monotones. Applications à des modèles élastoplastiques, Ph.D. thesis, Université Lyon I, Lyon, 2000, no. 962000.

[2] J. Bastien and M. Schatzman, Numerical precision for differential inclusions with uniqueness, M2AN Math. Model. Numer. Anal. 36 (2002), no. 3, 427-460.

[3] J. Bastien, M. Schatzman, and C.-H. Lamarque, Study of some rheological models with a finite number of degrees of freedom, Eur. J. Mech. A Solids 19 (2000), no. 2, 277-307.

[4] Study of an elastoplastic model with an infinite number of internal degrees of freedom, Eur. J. Mech. A Solids 21 (2002), no. 2, 199-222.

[5] H. Brézis, Opérateurs maximaux monotones et semi-groupes de contractions dans les espaces de Hilbert, North-Holland Mathematics Studies, no. 5. Notas de Matemática (50), NorthHolland, Amsterdam, 1973.

[6] B. Brogliato, Nonsmooth Impact Mechanics. Models, Dynamics and Control, Lecture Notes in Control and Information Sciences, vol. 220, Springer-Verlag, London, 1996.

[7] J. K. Hale and S. M. Verduyn Lunel, Introduction to Functional-Differential Equations, Applied Mathematical Sciences, vol. 99, Springer-Verlag, New York, 1993.

[8] C.-H. Lamarque, J. Bastien, and M. Holland, Study of a maximal monotone model with a delay term, SIAM J. Numer. Anal. 41 (2003), no. 4, 1286-1300.

[9] L. Paoli and M. Schatzman, A numerical scheme for impact problems. I. The one-dimensional case, SIAM J. Numer. Anal. 40 (2002), no. 2, 702-733.

[10] M. Schatzman, C.-H. Lamarque, and J. Bastien, An ill-posed mechanical problem with friction, Eur. J. Mech. A Solids 18 (1999), no. 3, 415-420.

Claude-Henri Lamarque: Laboratoire Géomatériaux, Département Génie Civil et Bâtiment, URA 1652 CNRS, École Nationale des Travaux Publics de l'Etat, rue Maurice-Audin, 69518 Vaulx-en-Velin Cedex, France

E-mail address: lamarque@entpe.fr

Jérôme Bastien: Laboratoire Mécatronique 3M, Équipe d'accueil A 3318, Université de Technologie de Belfort-Montbéliard, 90010 Belfort Cedex, France

E-mail address: jerome.bastien@utbm.fr

Matthieu Holland: CETE Normandie Centre, 76121 Grand-Quevilly Cedex, France 


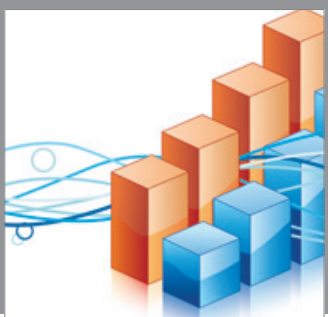

Advances in

Operations Research

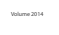

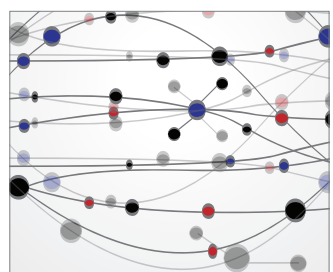

\section{The Scientific} World Journal
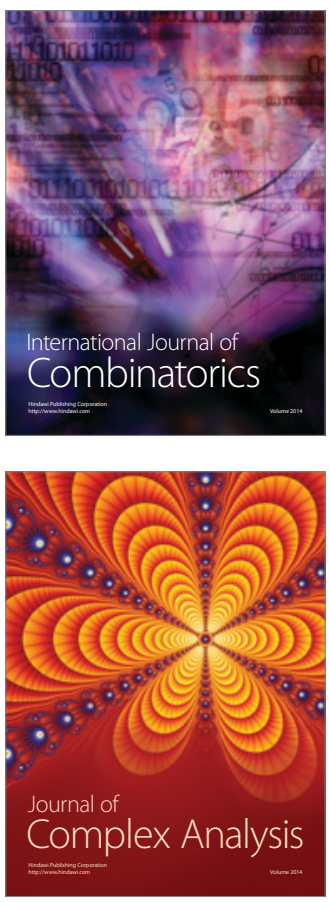

International Journal of

Mathematics and

Mathematical

Sciences
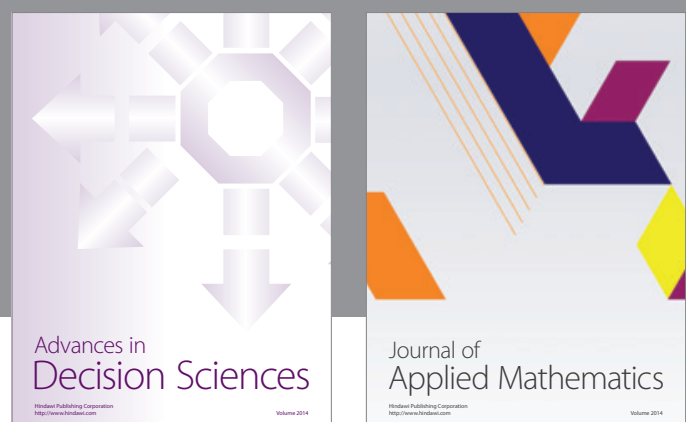

Journal of

Applied Mathematics
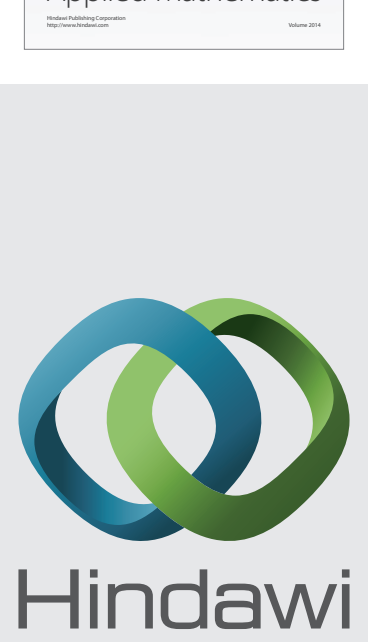

Submit your manuscripts at http://www.hindawi.com
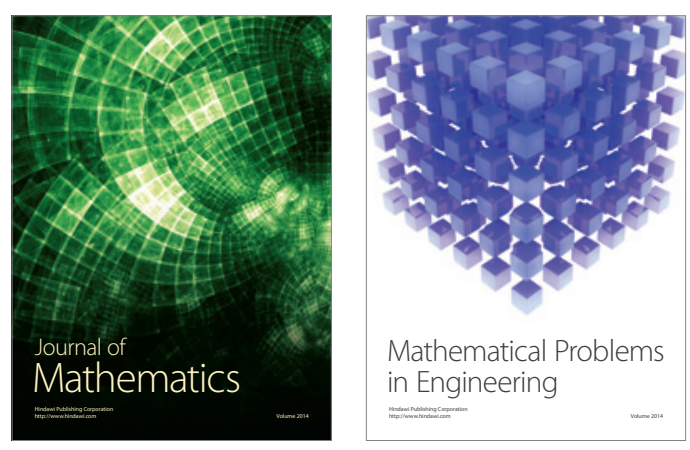

Mathematical Problems in Engineering
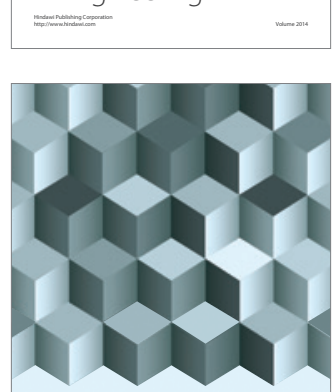

Journal of

Function Spaces
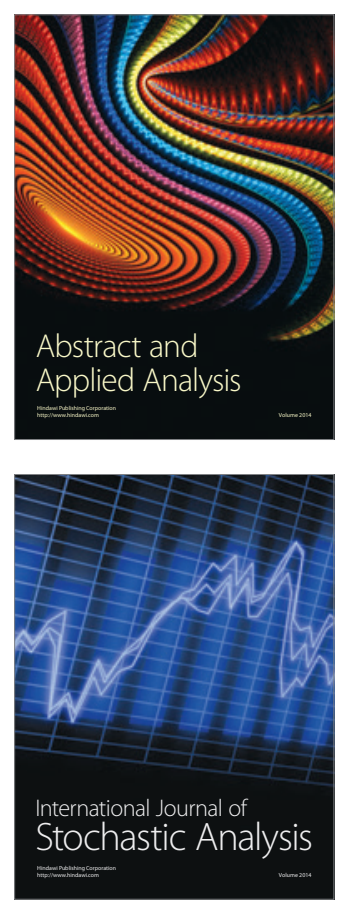

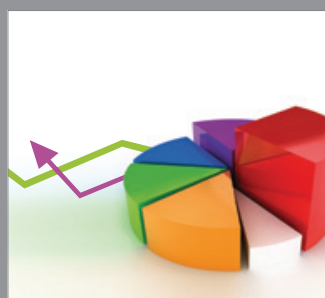

ournal of

Probability and Statistics

Promensencen
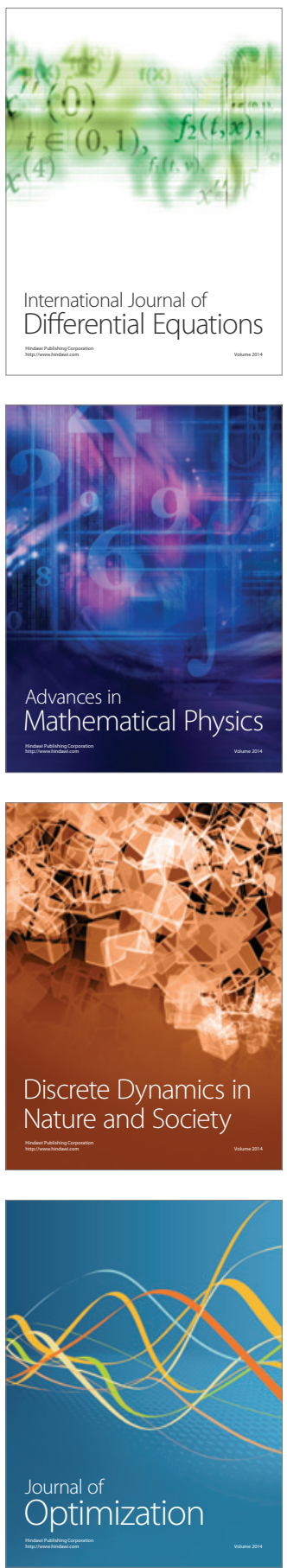